\title{
Temperature Sensors for Biochips Based on Resistance Temperature Detectors Using Copper Pattern on Printed Circuit Board
}

\author{
Sang-Yoon Kim, ${ }^{1,3}$ Jong-Dae Kim,,${ }^{2,3}$ Yu-Seop Kim, ${ }^{2,3}$ \\ Hye-Jeong Song, ${ }^{2,3}$ and Chan-Young Park ${ }^{2,3 *}$ \\ ${ }^{1}$ Department of Computer Engineering, Hallym University, \\ 1 Hallymdaehak-gil, Chuncheon, Ganwondo, South Korea \\ ${ }^{2}$ Department of Convergence Software, Hallym University, \\ 1 Hallymdaehak-gil, Chuncheon, Ganwondo, South Korea \\ ${ }^{3}$ Bio-IT Research Center, Hallym University, 1 Hallymdaehak-gil, Chuncheon, Ganwondo, South Korea
}

(Received March 1, 2015; accepted March 11, 2016)

Keywords: resistance temperature detector, metal thin film sensor, PCB pattern resistance

Biochips have been the center of attention in biological research because they can be employed in improving various genetic analysis techniques, such as reducing both analysis time and the quantity of sample required. In particular, biochips have been extensively employed in polymerase chain reaction (PCR) experiments, in which sensors are required to measure the temperature. This study proposed a temperature sensor chip that enables the implementation of a resistance temperature detector made of copper on a printed circuit board (PCB). The calibration of each sensor chip was investigated with either a 2-point or a 5-point fitting, and we determined that 2-point calibration was sufficient for application to PCR. The calibrated resistance detector chip can accurately measure the temperature at room temperature, $55,72,85$, and $95{ }^{\circ} \mathrm{C}$ in a water bath. The 2-point calibration resulted in less than a $0.52{ }^{\circ} \mathrm{C}$ error in the reported temperature.

\section{Introduction}

A biochip is a very useful tool that has been employed in much biological research and analysis. Biochips can be utilized for selectively and functionally fixing high-molecular biomaterials on surfaces and analyzing surface-bound biomaterials such as microarrays. It can also be used to analyze infinitesimal amounts of samples in a short time, which is highly advantageous in the polymerase chain reaction (PCR), which has been widely used in many studies since its invention by an American biochemist, Kary Mullis, in 1983. There have been many studies aimed at developing micro PCR chips for such applications.

PCR is a biomolecular technique that replicates and amplifies a very small amount of deoxyribonucleic acid (DNA) more than a hundredfold. ${ }^{(1,2)}$ This technique plays a significant role in broad areas associated with handling DNA, such as biochemistry, molecular biology, medicine, forensics for criminal investigation, and biological classification and analysis. PCR generally consists of the following three steps:

"Corresponding author: e-mail: cypark@hallym.ac.kr 
(1) DNA Denaturation: DNA is heated to $95^{\circ} \mathrm{C}$, and the double stranded DNA uncoils into two single strands of DNA.

(2) Primer Annealing: Primers mark the initial point of amplification, and two primers are attached to each strand. The attachment of primers to single stranded DNA and annealing occurs at $55-65^{\circ} \mathrm{C}$.

(3) DNA Extension: At $72{ }^{\circ} \mathrm{C}$, DNA polymerase extends the complementary strand of each single stranded DNA, completing a new pair of double stranded DNAs.

These three steps are one cycle in PCR, and this cycle is repeated multiple times, amplifying the target region of the DNA. In this procedure, Peltier and polyimide flexible heaters are used to increase the temperature for PCR systems using micro PCR chips, but the high unit prices of such heaters increases the overall cost of running a PCR. An earlier study was conducted by making a printed circuit board (PCB), which has a lower unit cost than the other materials mentioned, to serve as a substrate to integrate the heater and micro PCR chip platform. ${ }^{(3)}$ The PCB-based PCR chip requires temperature sensors to accurately measure the proper temperature at each step. Temperature sensors such as thermocouple sensors, thermistors, and resistance temperature detectors (RTD) can be installed on the PCB substrate, on the opposite side of the platform where the DNA is amplified.(4-8) Our group previously demonstrated successful DNA amplification using a PCB-based PCR chip with a thermistor having $1 \%$ resistance tolerance embedded into the PCB substrate. ${ }^{(9)}$ In the previous work, the PCR results from several PCR chips with thermistors having different error rates were statistically analyzed using the band volume of the gel image after amplification. The results showed that calibration of thermistors having different error rates was not necessary and did not affect the outcome of PCR amplification. Therefore, the copper pattern used as the heater in the PCB-based PCR chip, which can also serve as an RTD, might be able to replace the role of the thermistor serving as a temperature sensor. However, the error rate of the copper pattern used as an RTD should be as small as the thermistor employed as a temperature sensor. Therefore we investigated the error rate of the copper pattern used as an RTD by calibrating it. The calibration data can be gathered and recorded on the chip using a QR code, as demonstrated in our previous work. ${ }^{(9)}$

The resistance of metals, especially copper, is linear over a wide range of temperatures. ${ }^{(10)}$ Although the resistance of copper changes at temperatures over $150^{\circ} \mathrm{C}$ due to oxidation, this did not affect the present research on copper patterns as a temperature sensors since the highest temperature in the experiments was $98{ }^{\circ} \mathrm{C}$. The error rate of the temperature measured using a copper pattern as an RTD was analyzed according to calibration methods. The resistance of the copper pattern was measured at room temperature (20.6), 55, 72,85 , and $95^{\circ} \mathrm{C}$, all of which are critical temperatures in the PCR cycle. From the measured resistance, the temperature was calibrated with 2-point and 5-point fittings. The 2-point fitting uses only the end values of resistance that correspond to the room temperature and $95{ }^{\circ} \mathrm{C}$ when fitting the resistance to calibrate the temperature, and the 5-point calibration uses all 5 resistances measured. The difference between the temperature calibration using the 2-point and 5-point fitting was investigated and analyzed. The results showed that the 2-point fitting was sufficient to calibrate the temperature with high accuracy, having an error rate of less than $0.52{ }^{\circ} \mathrm{C}$. This indicated that the copper pattern on the PCB substrate is applicable as a temperature sensor for PCR applications. 


\section{Materials and Methods}

The resistance of the copper pattern, which increases linearly according to temperature, can be expressed as follows. ${ }^{(10)}$

$$
R(t)=R_{0}+A t,
$$

where $R(t), R_{0}, A$, and $t$ are the resistance of the copper pattern at temperature $t$, the resistance and the proportional coefficient for the calibration, and the temperature in ${ }^{\circ} \mathrm{C}$, respectively. The resistance at $0{ }^{\circ} \mathrm{C}, R_{0}$, varies chip-to-chip because the patterning in PCB production cannot deliver the ideal width for a limited cost. Therefore, $R_{0}$ should also be determined with the proportionality coefficient for calibration. Since the desired calibration equation should only have the resistance as an independent variable, Eq. (1) can be written as follows:

$$
t(R)=a R+b
$$

where $a$ and $b$ are the calibration coefficients to be determined.

Despite the calibration coefficients being more accurate as the number of measurement points increases, it is ideal to minimize the measurement points because of the cost. This study investigated the temperature error using a 2-point fitting with the end resistance values of room temperature and $95^{\circ} \mathrm{C}$, and a 5-point fitting using all resistance values at room temperature (20.6), $55,72,85$, and $95^{\circ} \mathrm{C}$. The temperature error is the difference between the calibration coefficients determined using the resistance and those determined when using actual temperatures in Eq. (2). For the 2-point fitting, the resistance values at 20.6 and $95{ }^{\circ} \mathrm{C}$ were used to fit the calibration coefficients and determine $a$ and $b$ with Eq. (2).

The structure of the chips and the copper pattern are shown in Fig. 1, where the copper pattern covers an area $15.0 \mu \mathrm{m}$ long and $10.0 \mathrm{~mm}$ wide. The copper pattern has a width of $100 \mathrm{~mm}$ and is separated by $100 \mu \mathrm{m}$ apart, having a total resistance of 5-7 $\Omega$ at room temperature. The QR code seen at the top of the left picture contains the Steinhart-Hart calibration coefficient for the thermistor seen in the middle of the right picture. This chip structure was used in the successful PCR amplification using the thermistor as the temperature sensor with a reaction chamber constructed on the opposite side of the pattern using tapes and films. ${ }^{(9)}$
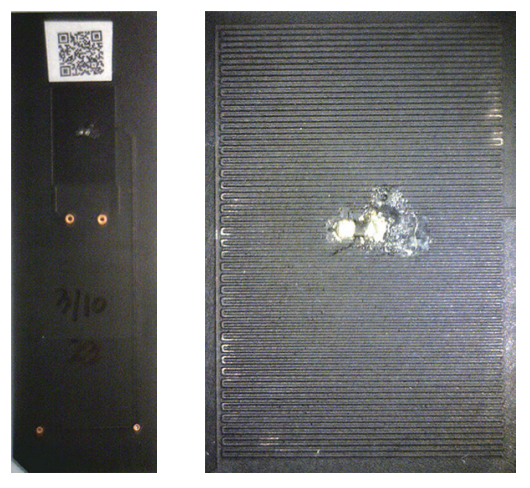

Fig. 1. (Color online) PCR chip and copper pattern. 
Measurements were obtained using a water bath (HETO OBN 8) and temperature control unit (HETO HMT 200) at temperatures of $20.6,55,72,85$, and $95{ }^{\circ} \mathrm{C}$, according to the temperature range used in PCR. Ten chips of the type shown in Fig. 1 were placed in the water bath at each temperature. In addition, a precision thermistor temperature sensor (US Sensor, USP3986) was placed in the water bath to verify the accuracy of the temperature displayed for the water bath. The chip pattern resistance and the precision thermistor were measured with two separate universal serial bus (USB) digital multimeters (NI USB-4065, National Instruments Corp.).

\section{Results}

The resistance values from the copper patterns as the RTDs of ten chips measured at each set temperature are shown in the 2 nd to 6 th columns of Table 1.

The calibration coefficients $a$ and $b$ derived from the 5-point fitting are listed in the 7th and 8 th columns in Table 1, followed by $1-R^{2}$, where $R^{2}$ is the coefficient of determination. Exponential notation was utilized for $1-R^{2}$ since $R^{2}$ was close to unity. The last two columns of Table 1 show the calibration coefficients obtained for resistances measured at 20.6 and $95{ }^{\circ} \mathrm{C}$, which correspond to the 2-point fitting. As can be seen in the table, the calibration coefficients obtained from 2-point and 5-point fittings have similar values.

The statistical differences between measured temperature and temperature calculated using Eq. (2) and the calibration coefficients obtained with either 5-point or 2-point fitting are shown in Table 2. The errors for 20.6 and $95{ }^{\circ} \mathrm{C}$ were excluded for the 2-point calibration because they were zero.

Table 1

Calibration coefficients of the chip RTD sensors.

\begin{tabular}{|c|c|c|c|c|c|c|c|c|c|c|}
\hline \multirow{3}{*}{ Chip No. } & \multicolumn{5}{|c|}{ Water bath temperature $\left({ }^{\circ} \mathrm{C}\right)$} & \multirow{2}{*}{\multicolumn{3}{|c|}{ 5-point fitting }} & \multirow{2}{*}{\multicolumn{2}{|c|}{ 2-point fitting }} \\
\hline & 20.6 & 55.0 & 72.0 & 85.0 & 95.0 & & & & & \\
\hline & \multicolumn{5}{|c|}{ Measured resistance $(\Omega)$} & $a$ & $b$ & $1-R^{2}$ & $a$ & $b$ \\
\hline 1 & 6.15 & 6.85 & 7.20 & 7.47 & 7.67 & 48.9 & -280.3 & $2.0 \mathrm{E}-05$ & 49.0 & -280.9 \\
\hline 2 & 5.95 & 6.63 & 6.97 & 7.23 & 7.42 & 50.3 & -278.8 & $3.2 \mathrm{E}-05$ & 50.5 & -279.5 \\
\hline 3 & 6.00 & 6.69 & 7.03 & 7.30 & 7.49 & 49.8 & -277.9 & $4.9 \mathrm{E}-05$ & 49.9 & -278.7 \\
\hline 4 & 5.27 & 5.86 & 6.16 & 6.38 & 6.55 & 58.2 & -286.0 & $3.2 \mathrm{E}-05$ & 58.3 & -286.6 \\
\hline 5 & 6.01 & 6.70 & 7.05 & 7.31 & 7.51 & 49.5 & -276.6 & $1.7 \mathrm{E}-05$ & 49.5 & -277.0 \\
\hline 6 & 5.05 & 5.62 & 5.90 & 6.12 & 6.28 & 60.5 & -285.2 & $2.0 \mathrm{E}-05$ & 60.6 & -285.8 \\
\hline 7 & 6.31 & 7.04 & 7.40 & 7.68 & 7.90 & 46.8 & -274.6 & $2.9 \mathrm{E}-05$ & 46.7 & -274.2 \\
\hline 8 & 5.19 & 5.77 & 6.06 & 6.28 & 6.45 & 58.9 & -285.2 & $1.8 \mathrm{E}-06$ & 59.0 & -285.3 \\
\hline 9 & 5.56 & 6.20 & 6.51 & 6.75 & 6.93 & 54.4 & -281.8 & $2.0 \mathrm{E}-05$ & 54.4 & -281.9 \\
\hline 10 & 5.39 & 6.00 & 6.29 & 6.52 & 6.70 & 56.8 & -285.3 & $5.7 \mathrm{E}-05$ & 56.6 & -284.4 \\
\hline
\end{tabular}

Table 2

Error statistics after 5-point and 2-point calibration.

\begin{tabular}{lcc}
\hline & \multicolumn{2}{c}{ Fitting error $\left({ }^{\circ} \mathrm{C}\right)$} \\
\cline { 2 - 3 } & 5 points & 2 points \\
\hline Min & -0.35 & -0.52 \\
Max & 0.24 & 0.37 \\
Mean & 0.00 & -0.06 \\
STD & 0.14 & 0.22 \\
\hline
\end{tabular}


The results showed that the maximum error in temperature using the 2-point fitting calibration was less than $0.52{ }^{\circ} \mathrm{C}$. Our previous work demonstrated that a $1{ }^{\circ} \mathrm{C}$ difference does not affect PCR performance, ${ }^{(9)}$ hence a 2-point calibration is sufficient when using a copper pattern on the PCB as a temperature sensor. However, to assure accuracy in temperature control, the measured resistance of the copper pattern should be precise, as well as accurate.

The sensitivity in regard to the resistance error is proportional to the calibration proportionality constant, which has a maximum value of 60 . Therefore, the resistance needs to be measured within $17 \mathrm{~m} \Omega(\Delta R=\Delta t / a)$ to assure that the temperature difference is less than $1{ }^{\circ} \mathrm{C}$. The digital multimeter used in this study has a resolution of $100 \mu \Omega$, which is much lower than the required resolution of $17 \mathrm{~m} \Omega$. Several tens of $\mathrm{m} \Omega$ resolution can also be obtained with a commercially available, low cost, low quality multimeter. The availability of commercial multimeters taken together with the calibration coefficient record through the QR code on the chip ${ }^{(10)}$ indicate the strong possibility of commercialization of a PCR chip-driving system with a PCB copper pattern temperature sensor.

Copper is easy to design and deposit in a pattern on a PCB. In addition, the copper pattern as an RTD sensor has good repeatability and reproducibility if it is used at temperatures below $150{ }^{\circ} \mathrm{C} \cdot\left({ }^{(10)}\right.$ Long-term tests for repeatability and reproducibility to guarantee long shelf-life of the chips remain to be carried out.

\section{Conclusions}

In this study, an RTD sensor designed and fabricated with copper on PCB for biochip temperature sensors was proposed. The error statistics of 2-point and 5-point calibration were investigated. The results indicated that 2-point calibration was sufficient for the temperature sensor for PCR chips. The temperature measurement system was qualitatively checked, suggesting the strong possibility of employing the $\mathrm{Cu}$ pattern RTD sensor as a PCR chip-driving system. A system with an accuracy of $10 \mathrm{~m} \Omega$ will be sufficient for PCR applications.

PCR chips with heating and sensing using PCB patterns only are more feasible, but further studies on the long term stability of the repeatability and reproducibility must be conducted.

\section{Acknowledgements}

This research was supported by the Basic Science Research Program of the National Research Foundation of Korea (NRF), which is funded by the Ministry of Education, Science and Technology (2012R1A1A2040381), by the Research and Business Development 2013 project of the Ministry of Trade, Industry and Energy (N0000907), and by Hallym University Research Fund, 2015 (HRF201512-009).

\section{References}

1 J. M. Bartlett and D. Stirling: A Short History of the Polymerase Chain Reaction, ed. Springer (PCR protocols, New York City, 2003) pp. 3-6, p. 226.

2 K. B. Mullis, H. A. Erlich, N. Arnheim, G. T. Horn, R. K. Saiki, and S. J. Scharf: U.S. Patent No. 4,683,195 (1987).

3 J. S. Hwang, S. Y. Kim, Y. S. Kim, H. J. Song, C. Y. Park, and J. D. Kim: Int. J. Control Autom. 8 (2015) 117.

4 J. G. Lee, K. H. Cheong, N. Huh, S. Kim, J. W. Choi, and C. Ko: Lab Chip 6 (2006) 886. 
5 E. Salm, Y. S. Liu, D. Marchwiany, D. Morisette, Y. He, A. K. Bhunia, and R. Bashir: Biomed. Microdevices 13 (2011) 973.

6 J. Wu, R. Kodzius, K. Xiao, J. Qin, and W. Wen: Biomed. Microdevices 14 (2012) 179.

7 C. Koo, M. Malapi-Wight, H. S. Kim, O. S. Cifci, V. L. Vaughn-Diaz, B. Ma, S. Kim, H. Abdel-Raziq, K. Ong, and Y. K. Jo: PloS One 8 (2013) e82704.

8 C. Y. Park, J. D. Kim, J. H. Ku, Y. S. Kim, H. J. Song, and J. Kim: Sens. Lett. 10 (2012) 1197.

9 S. B. N. Koo, J. H. Cho, Y. S. Kim, H. J. Song, C. Y. Park, and J. D. Kim: Int. J. Control Autom. 8 (2015) 149 .

10 The Measurement, Instrumentation, and Sensors Handbook, ed. J. G. Webster (CRC Press LLC, USA, 1999) pp. 32.13-32.25. 